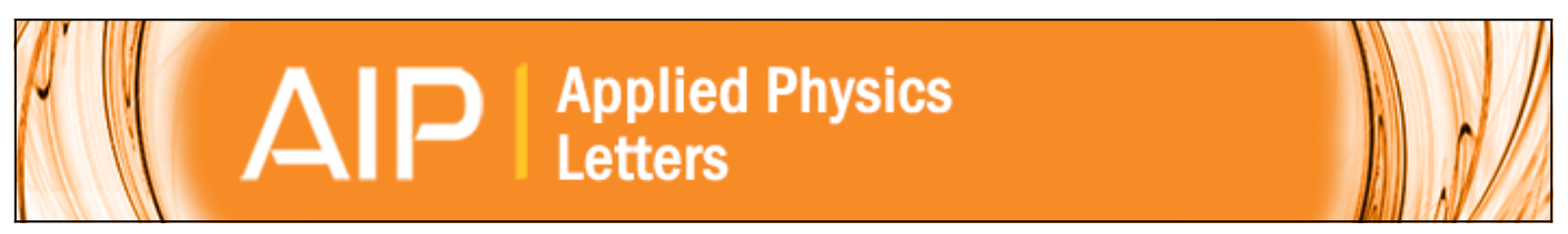

Detailed balance analysis of area de-coupled double tandem photovoltaic modules

Rune Strandberg

Citation: Applied Physics Letters 106, 033902 (2015); doi: 10.1063/1.4906602

View online: http://dx.doi.org/10.1063/1.4906602

View Table of Contents: http://scitation.aip.org/content/aip/journal/apl/106/3?ver=pdfcov

Published by the AIP Publishing

High-Voltage Amplifiers

- Voltage Range from $\pm 50 \mathrm{~V}$ to $\pm 60 \mathrm{kV}$

- Current to $25 \mathrm{~A}$

Electrostatic Voltmeters

- Contacting \& Non-contacting

- Sensitive to $1 \mathrm{mV}$

- Measure to $20 \mathrm{kV}$
ENABLING RESEARCH AND

INNOVATION IN DIELECTRICS,

ELECTROSTATICS,

MATERIALS, PLASMAS AND PIEZOS

TRek www.trekinc.com 


\title{
Detailed balance analysis of area de-coupled double tandem photovoltaic modules
}

\author{
Rune Strandberg ${ }^{\text {a) }}$ \\ Department of Engineering Sciences, University of Agder, Jon Lilletuns vei 9, No-4879 Grimstad, Norway
}

(Received 9 December 2014; accepted 14 January 2015; published online 22 January 2015)

\begin{abstract}
This paper describes how layers of area de-coupled top and bottom cells in photovoltaic tandem modules can increase the efficiency of two-terminal tandem devices. The point of the area de-coupling is to allow the number of top cells to differ from the number of bottom cells. Within each of the layers, the cells can be horizontally series-connected and the layers can then be currentor voltage-matched with each other in a tandem module. Using detailed balance modeling, it is shown that two-terminal tandem modules of this type can achieve the same theoretical efficiency as stacks of independently operated cells, often referred to as four-terminal cells. Optimal ratios of the number of bottom cells to the number of top cells are calculated. Finally, it is shown that modules with a bottom layer consisting of 60 cells with a band gap of $1.11 \mathrm{eV}$, resembling standard silicon modules, offer sufficient resolution to optimize the number of top cells and achieve high efficiency over a large range of top cell band gaps. This result extends the list of materials that can be used as top cells in two-terminal tandem modules with silicon bottom cells. (C) 2015 AIP Publishing LLC. [http://dx.doi.org/10.1063/1.4906602]
\end{abstract}

Photovoltaic tandem cells consist of a stack of two or more solar cells. The band gap of the active material of the cells should decrease from top to bottom in the stack. This allows tandem cells to convert more of the solar energy to electricity than conventional single band gap cells. ${ }^{1}$ Theoretical efficiencies of tandem cells can be calculated for a plethora of conditions and by applying a variety of assumptions and models. ${ }^{2}$ The most widely used method to compare photovoltaic concepts is the detailed balance formalism. This was first applied by Shockley and Queisser ${ }^{3}$ in their analysis of the single band gap cell, and later by de Vos in a similar analysis of tandem cells. ${ }^{4}$ The detailed balance formalism is also used in the present work. Important varieties of the detailed balance model regard the way the cells in the stack are connected. The two cells in a double tandem stack can be series-connected or operated independently. A seriesconnected stack is a two-terminal device, where the current produced by the top and bottom cells has to match. In an unconstrained stack, the cells are operated independently and current-matching is not required. Unconstrained stacks have a slightly higher limiting efficiency under the AM1.5G spectrum than the series-connected cells, but the difference is small with $46.1 \%$ versus $45.7 \% .^{5}$ A drawback of unconstrained stacks is the need for more than two terminals, which increases the complexity of the structure and introduces a clear practical disadvantage since commercially available cables, connections, and inverters for PV-systems are designed for two-terminal systems.

In Ref. 1, a distinction is made between tandem stacks with and without low-pass reflectors between the cells. In a double tandem stack, there will be one such reflector, whose purpose is to reflect luminescence from the top cell back to the top cell. This reduces the thermalization losses that occur when luminescent photons from the top cell are absorbed by

a)runes@uia.no the bottom cell. For photon energies lower than the band gap of the top cell, the reflector is assumed to be transparent. The use of such low-pass reflectors slightly increases the limiting efficiency of tandem cells, but only by $0.2 \%-0.3 \%$ absolute. ${ }^{1}$ Low-pass reflectors are assumed in all calculations to be presented in this paper.

An important drawback of series-connected stacks is a more rapid decline of the theoretical efficiency as the combination of band gaps moves away from the optimal. For reference, a plot of the theoretical efficiency of both seriesconnected and unconstrained double tandem cells under the AM1.5G spectrum is plotted in Figure 1. The calculation of these efficiencies follows the work of Martí and Araújo ${ }^{1}$ and Brown and Green, ${ }^{6}$ but using the most recent AM1.5G spectrum (ASTM G-173-03), which was also used by Bremner et al. ${ }^{5}$ for similar calculations.

For series-connected cells, the detailed balance efficiency for certain combinations of sub-optimal band gaps can be improved if some high-energy photons are transmitted to the bottom cell. This can be achieved by omitting the low-pass reflector and reducing the thickness of the top cell, ${ }^{7}$ or by reducing the area of the top cell to expose parts of the bottom cell to full-spectrum sunlight. ${ }^{8}$ Due to increased thermalization losses, neither of these methods can result in an efficiency equal to the efficiency of an unconstrained stack, unless the voltage of the top cell is lower than or equal to that of the bottom cell. But if the voltage of the top cell is not larger than that of the bottom cell, there is no advantage of having a tandem structure over a single band gap cell. And in any tandem cell where the top cell is operated at a larger voltage than the bottom cell, the energy of high energy photons is best utilized when being absorbed by the top cell.

The contribution of the present work is to simulate a double tandem module where the top cells and bottom cells are horizontally series-connected, instead of the vertical series-connection that has been analyzed in previous work. 
(a)

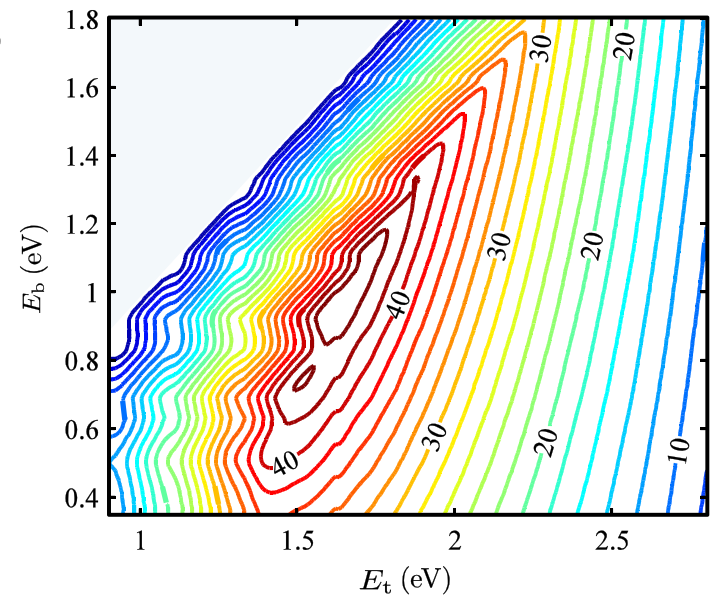

(b)

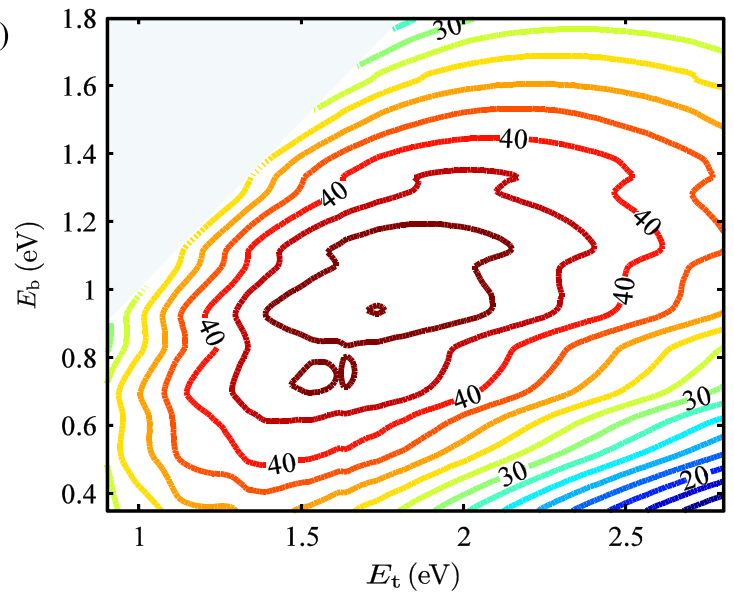

FIG. 1. Theoretical efficiency of double tandem cells illuminated by the AM1.5G spectrum as a function of the band gap of the top cell, $E_{\mathrm{t}}$, and the band gap of the bottom cell, $E_{\mathrm{b}}$. Plots are shown for (a) series-connected two-terminal cells, and (b) independently operated cells. The figures are calculated using methods from Refs. 1 and 6.

Horizontal series-connection allows the number of top cells to differ from the number of bottom cells while maintaining equal total top cell and total bottom cell areas. The resulting area de-coupling allows current-matching of the layers by adjusting the number of top cells and bottom cells independently. The horizontal series-connection also allows the two layers to be voltage-matched if connected in parallel as shown in Figure 2. If a module can be divided into an

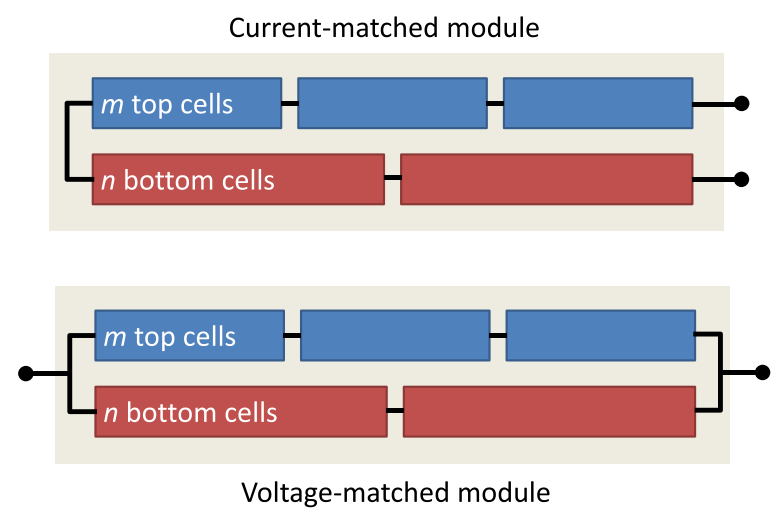

FIG. 2. Sketch of area de-coupled double tandem modules with horizontal series-connection. The upper module is current-matched and the lower module is voltage-matched. unlimited number of cells, perfect current or voltagematching can be achieved by fine-tuning the ratio of the number of bottom cells to the number of top cells. The cells in both layers are then operated at their maximum efficiency point, which means that the efficiency of such a two-terminal module equals that of a stack of independently operated cells.

The optimal ratio of the number of bottom cells $n$ to the number of top cells $m$ depends on the incoming spectrum, as well as the two band gaps, and can be calculated using a detailed balance model. In the following, it is assumed that both top and bottom cells are completely absorbing photons with energy higher than the respective band gaps and completely transparent for photons with lower energy. It is also assumed that there is a perfect reflector on the back side of the bottom cells and that there is an ideal low-pass reflector between the layers of cells. Charge carriers are assumed to have infinite mobility, which implies that the quasi-Fermi levels are constant throughout each cell. It is also assumed that all recombination is radiative and that there are no reflective losses. The flux of photons with energy between $E_{\mathrm{a}}$ and $E_{\mathrm{b}}$ emitted by a solar cell biased with a voltage $V$ and obeying the above list of assumptions is given by ${ }^{9}$

$$
\phi_{\text {out }}\left(E_{\mathrm{a}}, E_{\mathrm{b}}, V\right)=C \int_{E_{\mathrm{a}}}^{E_{\mathrm{b}}} \frac{E^{2}}{\exp [(E-q V) / k T]-1} \mathrm{~d} E,
$$

where $T$ is the temperature of the cell and set to $300 \mathrm{~K}$ in this work. The constant $C$ equals $2 \pi /\left(h^{3} c^{2}\right)$, where $h$ is Planck's constant, $c$ is the speed of light, and $k$ is Boltzmann's constant.

The core of detailed balance modeling is that the number of electrons delivered to an external circuit by a solar cell equals the net number of photons absorbed by the cell. Having a double tandem module with $m$ series-connected top cells and a total module area $A$, the top layer will deliver a current

$$
I_{\mathrm{t}}=\frac{q A}{m}\left(\phi_{\text {in }}\left(E_{\mathrm{t}}, \infty\right)-\phi_{\text {out }}\left(E_{\mathrm{t}}, \infty, V_{\mathrm{t}}\right)\right)
$$

where $q$ is the elementary charge, $E_{\mathrm{t}}$ is the band gap of the top cell, and $V_{\mathrm{t}}$ is the voltage of each cell in the top layer. $\phi_{\text {in }}\left(E_{\mathrm{t}}, \infty\right)$ is the incoming flux of photons with energy between $E_{\mathrm{t}}$ and infinity. The latter is calculated from the solar spectrum, and, as mentioned above, the AM1.5G spectrum is used in this work.

For a module with $n$ series-connected bottom cells, the current delivered by the bottom layer is given by

$$
I_{\mathrm{b}}=\frac{q A}{n}\left(\phi_{\text {in }}\left(E_{\mathrm{b}}, E_{\mathrm{t}}\right)-\phi_{\text {out }}\left(E_{\mathrm{b}}, E_{\mathrm{t}}, V_{\mathrm{b}}\right)\right),
$$

where $E_{\mathrm{b}}$ is the band gap of the bottom cells and $V_{\mathrm{b}}$ is the voltage of each cell in the bottom layer.

If the two layers of cells are connected in series, the current-matching implies that $I_{\mathrm{t}}=I_{\mathrm{b}}=I$. If they are connected in parallel and voltage-matched, the relation $m V_{\mathrm{t}}=n V_{\mathrm{b}}=V$ must be obeyed.

The total power produced by the module is then given by

$$
P_{\mathrm{s}}=\left(m V_{\mathrm{t}}+n V_{\mathrm{b}}\right) I
$$


with series-connected layers, and

$$
P_{\mathrm{p}}=V\left(I_{\mathrm{t}}+I_{\mathrm{b}}\right) \text {, }
$$

when the layers are connected in parallel. Dividing the output power by the module area and the power density of the AM1.5G spectrum gives the module efficiency.

Plots of the optimal ratio of the number of bottom cells to the number of top cells $(n / m)$ are shown in Figure 3 for both current- and voltage-matched-modules. The efficiency of cells with optimized $n / m$-ratio corresponds to that of a stack of independently operated cells shown in Figure 1(b). Note that the iso-line with $n / m=1$ in Figure 3(a) closely follows the high-efficiency ridge in Figure 1(a). Efficiency maps calculated with constant values of the $n / m$-ratio similarly show narrow ridges close to the corresponding iso-lines.

A typical PV module of today consists of 60 silicon cells. Figure 4 shows the optimal number of top cells in tandem modules consisting of 60 bottoms cells with a band gap of $1.11 \mathrm{eV}$. The values are plotted as a function of the top cell band gap. In current-matched modules, a large number of top cells are required when $E_{\mathrm{t}}$ is small because the bottom cell then receives few photons. The current density generated by the cells in the top layer is then much higher than the current density generated by the cells in the bottom layer. As the band gap of the top cells increases, the flux of photons reaching the bottom cells grows while the flux of photons absorbed by the top layer decreases. Consequently, the optimal number of top cells decreases with the magnitude of $E_{\mathrm{t}}$. In voltage-matched modules, the number of top cells is lower

(a)

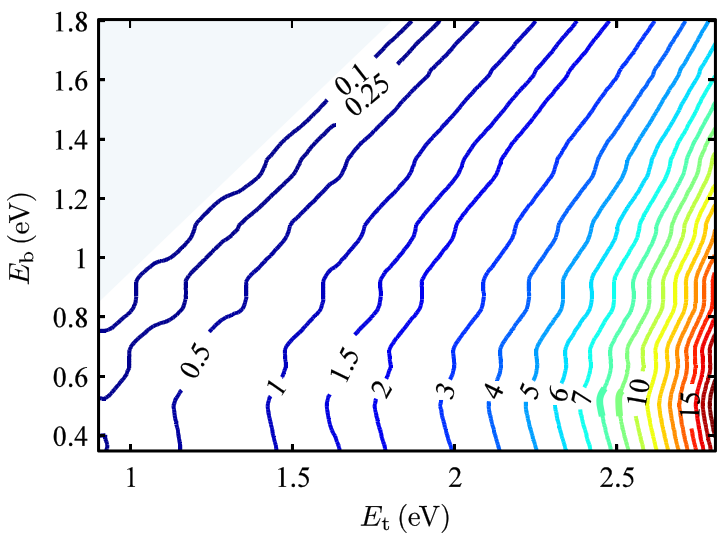

(b)

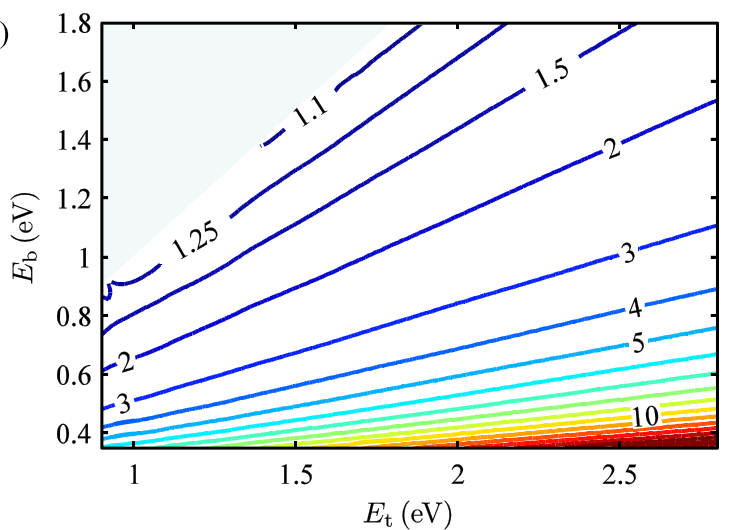

FIG. 3. (a) The optimal number of bottom cells to the number of top cells $(n / m)$ in a current-matched module. (b) The same ratio for a voltagematched module.
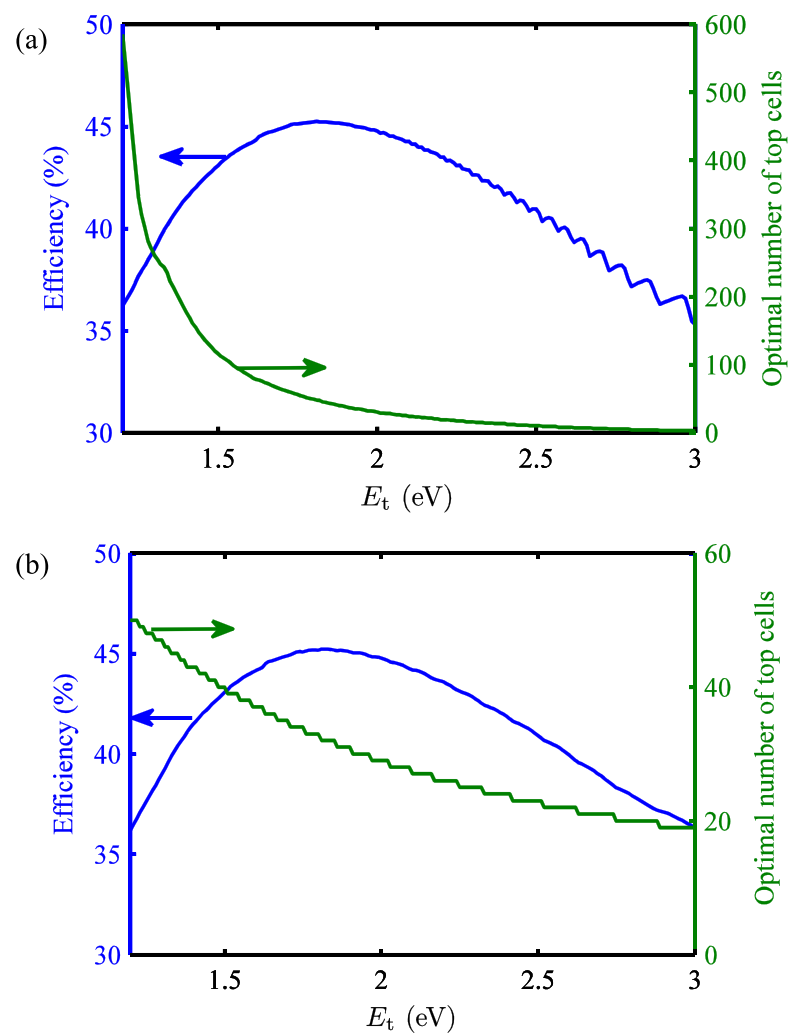

FIG. 4. The optimal number of top cells on a module consisting of 60 bottom cells with a band gap of $1.11 \mathrm{eV}$. Plots are shown for current-matched modules (a) and voltage-matched modules (b). The calculations are carried out with steps of $0.01 \mathrm{eV}$ along the horizontal axis. Both graphs also show the theoretical efficiency of the respective modules.

than the number of bottom cells, because of the larger band gap, and thus higher voltage, of each top cell. As $E_{\mathrm{t}}$ increases, so does the difference in voltage, and the optimal value of $n / m$ decreases.

The efficiency obtained by optimized 60 bottom-cell modules is also plotted in Figure 4. The maximal efficiency of both module types equals the $45.2 \%$ limit of an independently operated stack with a bottom-cell band gap of $1.11 \mathrm{eV}$. The optimal current-matched module has 48 top cells with a band gap of $1.81 \mathrm{eV}$, while the optimal voltage-matched module has 32 top cells with a band gap of $1.82 \mathrm{eV}$. A voltage-matched module shows an efficiency above $40 \%$ for top-cell band gaps between 1.34 and $2.59 \mathrm{eV}$. Figure 4(b) shows that the efficiency of current-matched modules with a large top-cell band gap starts to oscillate with increasing $E_{\mathrm{t}}$. For large values of $E_{\mathrm{t}}$, the ideal number of top cells eventually drops below 10, which is limiting the resolution available when optimizing the $n / m$-ratio. The amplitude of the oscillations can be reduced if the number of bottom cells is increased, for example, to 72-which is already the number of cells in some commercial modules.

In a recent work by White et al., ${ }^{10}$ a selection of materials are identified as promising materials for realizing efficient modules based on earth-abundant thin film cells in tandem with silicon cells. In the cited work, they assume four-terminal tandem stacks with independently operated cells. Taking into account the concept of current- or voltagematching by area de-coupling, the results obtained by White et al. are also valid for two-terminal modules. Thin-film 
deposition gives great freedom in dividing modules into various numbers of cells. Depositing thin-film cells on the front glass of a silicon module might allow area de-coupled tandem modules to be produced while keeping many of the steps in existing production lines for wafer-based silicon modules unchanged.

It should be observed that the results presented above are obtained using a fixed spectrum and a constant cell temperature. During outdoor operation, PV modules inevitably experience varying conditions. When the conditions shift, the current- or voltage-matching of the modules will be affected. In an independently operated tandem stack, the voltage of the different cell types can be kept at the maximum power point continuously. In general, this is not the case for modules that are current- or voltage-matched by area de-coupling, because the areas of the different cells are fixed and optimized for a particular set of conditions. This gives independently operated stacks an advantage over area de-coupled two-terminal modules.

To summarize, this article explains how the theoretical efficiency of two-terminal double tandem modules can match that of independently operated double tandem stacks, by area de-coupling of the cells in the top and bottom layer. The optimal ratio of the number of bottom cells to the number of top cells is calculated for modules where the top and the bottom layers are either current- or voltage-matched. It is then shown that modules with a bottom layer of 60 cells with a band gap of $1.11 \mathrm{eV}$ in most cases offers sufficient resolution to properly optimize the $n / m$-ratio of the module. Exceptions are found in current-matched modules with a large top-cell band gap.

The author would like to thank the PV group at the University of Agder for valuable feedback and discussions on this work.

${ }^{1}$ A. Martí and G. Araújo, Sol. Energy Mater. Sol. Cells 43, 203 (1996).

${ }^{2}$ S. Kurtz, D. Myers, W. E. McMahon, J. Geisz, and M. Steiner, Prog. Photovoltaics 16, 537 (2008).

${ }^{3}$ W. Shockley and H. J. Queisser, J. Appl. Phys. 32, 510 (1961).

${ }^{4}$ A. de Vos, J. Phys. D: Appl. Phys. 13, 839 (1980).

${ }^{5}$ S. P. Bremner, M. Y. Levy, and C. B. Honsberg, Prog. Photovoltaics 16, 225 (2008).

${ }^{6}$ A. S. Brown and M. A. Green, Prog. Photovoltaics 10, 299 (2002).

${ }^{7}$ S. R. Kurtz, P. Faine, and J. M. Olson, J. Appl. Phys. 68, 1890 (1990).

${ }^{8}$ J. Yang, D. Cheong, J. Rideout, S. Tavakoli, and R. Kleiman, in Proceedings of the 37th IEEE Photovoltaic Specialists Conference (IEEE, 2011), pp. 1019-1024.

${ }^{9}$ A. Vos and H. Pauwels, Appl. Phys. 25, 119 (1981).

${ }^{10}$ T. White, N. Lal, and K. Catchpole, IEEE J. Photovoltaics 4, 208 (2014). 